\title{
Isostrychnopentamine, an indolomonoterpenic alkaloid from Strychnos usambarensis, with potential anti-tumor activity against apoptosis-resistant cancer cells
}

\author{
EL-HADJ SAIDOU BALDE ${ }^{1}$, VÉRONIQUE MÉGALIZZI ${ }^{1}$, MARTINE CAO $^{2}$, LUC ANGENOT $^{2}$, \\ ROBERT KISS $^{1}$, MARC VAN DAMME ${ }^{1}$ and MICHEL FREDERICH ${ }^{2}$ \\ ${ }^{1}$ Laboratoire de Toxicologie, Département de BioAnalyse et Toxicologie Pharmaceutique, Institut de Pharmacie, \\ Université Libre de Bruxelles (ULB), Brussels; ${ }^{2}$ Laboratory of Pharmacognosy, \\ Drug Research Center (CIRM), Université de Liège, Liège, Belgium
}

Received September 8, 2009; Accepted October 26, 2009

DOI: 10.3892/ijo_00000575

\begin{abstract}
Isostrychnopentamine (ISP) is an indolomonoterpenic alkaloid that is present in the leaves of Strychnos usambarensis, an East African small tree. We have reported previously pro-apoptotic effects induced in vitro by ISP in the human HCT-116 colon cancer cell line, a model that displays relative sensitivity to apoptosis. In the present study, we observed that the in vitro growth inhibitory activities of ISP are similar in cancer cells that display sensitivity versus resistance to apoptosis. We made use of the U373 glioblastoma and the A549 non-small cell lung cancer (NSCLC) cell lines as models relatively resistant to apoptosis, and the human PC-3 prostate cancer cell line as a model relatively sensitive to apoptosis. While ISP induced transient decreases in [ATP] and apoptosis in human U373 GBM cells, it did not provoke such features in A549 NSCLC cells. It thus seems that ISPinduced anti-cancer activity can lead to pro-apoptotic effects as a consequence, while apoptosis seems not to be the main cause by which ISP induces cancer cell death. ISP is a compound that merits further investigations in order to: i) identify the mechanism(s) of action by which it kills cancer cells, and ii) hemisynthesize novel ISP derivatives aiming to overcome, at least partly, the resistance of metastatic cancers to apoptosis.
\end{abstract}

\section{Introduction}

Despite frequent responses to chemotherapy, curative treatment remains elusive for the majority of patients with metastatic

Correspondence to: Dr Robert Kiss, Laboratory of Toxicology, Insitute of Pharmacy, Université Libre de Bruxelles, Campus de la Plaine, CP205/1, Boulevard du Triomphe, 1050 Brussels, Belgium E-mail: rkiss@ulb.ac.be

Abbreviations: ATP, adenosine triphosphate; GBM, glioblastoma; ISP, isostrychnopentamine; NSCLC, non-small cell lung cancer; SEM, standard error on the mean; WHO, World Health Organization

Key words: cancer, apoptosis, resistance, isostrychnopentamine solid tumors (1). Indeed, in the common advanced cancers, over 40 years of cytotoxic drug development has brought no significant change in cure rates (1). Savage and colleagues (1) report that one interpretation is that the intrinsic properties of the malignancies themselves, rather than the qualities of individual drugs or combination therapies, are primarily responsible for their curability with chemotherapy. These authors thus suggest that the curability of these malignancies results from an intrinsic 'locked-in' state of sensitivity to proapoptotic stresses in these cells (1). In fact, $>90 \%$ of cancer patients die from their metastases (2). Drug resistance, either acquired or intrinsic, often prevents tumor cells from undergoing sufficient levels of apoptosis, resulting in cancer cell survival and treatment failure $(1,2)$. The metastatic process is inherent to a significant level of resistance to apoptosis. Indeed, as a barrier to metastases, cells normally undergo apoptosis after they lose contact with their extra cellular matrix or their neighboring cells (3). This cell death process has been termed 'anoikis' (3). Tumor cells that acquire malignant potential have developed mechanisms to resist anoikis and thereby survive after detachment from their primary site and while travelling through the lymphatic and circulatory systems (3). Defects in the death receptor pathway of caspase activation, such as the over-expression of the caspase- 8 inhibitor FLIP, can render cells resistant to ano1kis (3).

Plants have played a dominant role in the development of sophisticated traditional medicine systems (4). The continuing and essential role played by plant-based systems in the healthcare of many different cultures has been extensively documented (4) and the WHO has estimated that approximately $65 \%$ of the world's population relies mainly on plant-derived traditional medicines for their primary health care (4). Plants have a long history of use in the treatment of cancer (4). However, while plant-derived compounds display an amazingly broad range of mechanisms of action when exerting anticancer effects (4), only few compounds activate apoptosis in transformed cells (4). B-lapachone, a close relative of Blapachol obtained from the bark of the lapacho tree, Tabebuia avellanedae, induces apoptosis in transformed cells through activation of caspase-3, inhibition of NF-кB, and subsequent down-regulation of bcl-2 (4). Currently, B-lapachone 
(ARQ501) is in phase II clinical trials in the USA for advanced solid tumors (4).

We have studied for several years the in vitro antimalarial activities of bisindole alkaloids from Strychnos species (5) and we have identified from Strychnos usambarensis, a small African tree, isostrychnopentamine (ISP) $(6,7)$ as a compound possessing very potent antimalarial properties (8). This compound was also able to induce apoptosis in human colon cancer cells through activation of caspase-3 and -9 (9). In the present study we have investigated the ability of ISP in inducing apoptosis in vitro in two types of apoptosis-resistant cancers, i.e., glioblastoma (GBM) (10-12) and non-small cell lung cancer (NSCLC) $(13,14)$. We used as a positive control the human $\mathrm{PC}-3$ prostate cancer cell line in which plantderived compounds, such as narciclasine (15) or sodium pump inhibitors (16) are able to induce apoptosis in vitro. Narciclasine and sodium pump inhibitors are not able to induce apoptosis in vitro in the U373 GBM $(17,18)$ and A549 NSCLC (19) models: these two models therefore display a certain level of resistance to apoptosis.

\section{Materials and methods}

Cell cultures and compounds. The human U373 GBM [ECACC (European Collection of Cell Culture; Salisbury, UK) code 89081403], A549 NSCLC [DSMZ (Deutsche Sammlung von Mikroorganismen and Zellkulturen; Braunschweig, Germany) code ACCC107], PC-3 prostate cancer (DSMZ code ACC465) and MCF-7 breast cancer (DSMZ code ACC115) were cultured as detailed elsewhere $(15,17,18)$. ISP was obtained as described elsewhere (9); its chemical structure is illustrated in Fig. 1.

ISP in vitro growth inhibitory activity. The overall growth level of human U373, A549 and PC-3 cancer cells was determined by means of the MTT colorimetric assay as detailed elsewhere $(15,17,18)$. The cancer cells were cultured for 3 days in the presence (or absence as a control) of ISP concentrations ranging from $1 \mathrm{nM}$ to $10 \mu \mathrm{M}$. The experiments were performed in hexaplicate. These experiments have enabled the ISP $50 \%$ growth inhibitory concentration $\left(\mathrm{IC}_{50}\right)$ to be determined for each of the three human cancer cell lines under study.

In vitro computer-assisted phase-contrast microscopy analyses of ISP anti-cancer effects. The in vitro anti-cancer effects of ISP at its $\mathrm{IC}_{50}$ concentration (PC-3 cancer cells) or at 5-fold its $\mathrm{IC}_{50}$ concentration (U373 and A549 cancer cells) were visualized by means of computer-assisted phase-contrast microscopy, i.e., quantitative videomicroscopy (20,21). Cells were monitored for $72 \mathrm{~h}$. 'Movies' were built on the obtained time-lapse image sequences and enabled a rapid screening for cell viability $(20,21)$. In each control or ISP-treated condition, the cell growth level was evaluated by the ratio between the numbers of cells counted in the last and first frames of the image sequences $(21,22)$. All the cell counts were performed in triplicate using an interactive computer tool $(21,22)$.

In vitro apoptosis and necrosis determination. Apoptosis and necrosis were determined using flow cytometry analyses of

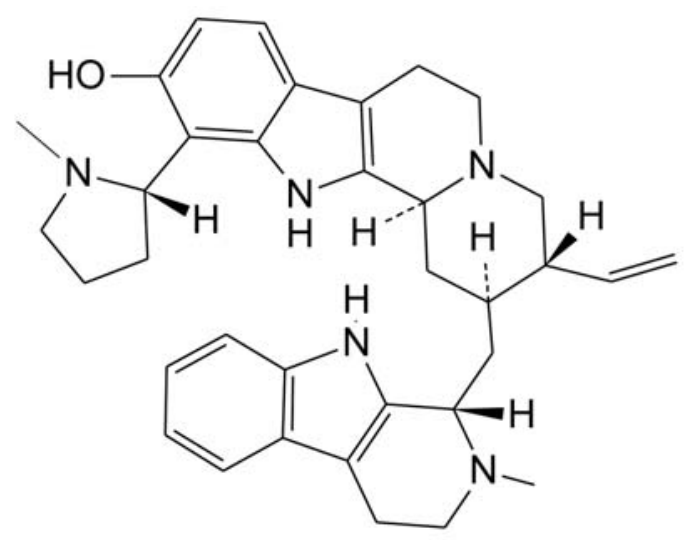

Figure 1. Chemical structure of isostrychnopentamine (ISP), which is an indolomonoterpenic alkaloid from Strychnos usambarensis.

Annexin V/propidium iodide double staining, as detailed elsewhere $(15,18,19,23)$. Experiments were performed in triplicate.

Cellular ATP $\left([\mathrm{ATP}]_{i}\right)$ measurements. $[\mathrm{ATP}]_{\mathrm{i}}$ was measured using a bioluminescence assay (Invitrogen, Merelbeke, Belgium) in U373 GBM and A549 NSCLC cells cultured in the presence (or absence as a control) of ISP at its $\mathrm{IC}_{50}$ concentrations $(\sim 1 \mu \mathrm{M})$, as detailed elsewhere $(17,23)$. Three replicate determinations per time point were performed for each cell line.

Statistical analyses. Statistical analyses were performed using Statistica software (Statsoft, Tulsa, USA). Statistical comparison of control and treated groups was undertaken with the Mann-Whitney U test (a non-parametric one-way analysis of variance for two groups).

\section{Results}

Determination of ISP IC ${ }_{50}$ in vitro growth inhibitory concentrations in human cancer cells. Fig. 2A shows that ISP $\mathrm{IC}_{50}$ concentrations equal $\sim 1 \mu \mathrm{M}$ in $\mathrm{U} 373 \mathrm{GBM}$ and $\mathrm{A} 549$ NSCLC cells and $\sim 3 \mu \mathrm{M}$ in PC-3 prostate cancer cells. Thus, U373 GBM (18) and A549 NSCLC (19) cells, which are associated with certain levels of resistance to apoptosis, are as sensitive to ISP as PC-3 prostate cancer cells that are sensitive to apoptosis (15).

The data in Fig. 2B (obtained by means of quantitative videomicroscopy) validate those illustrated in Fig. 2A (obtained by means of the MTT colorimetric assay) and clearly indicate that ISP displays marked anti-tumor effects. Indeed, increasing by 5 -fold the ISP $\mathrm{IC}_{50}$ concentration [from 0.8 (Fig. 2A) to 4 (Fig. 2B) $\mu \mathrm{M}$ ] completely prevented U373 GBM and A549 NSCLC cell population growth after 12 and $24 \mathrm{~h}$ of ISP addition in the culture media, respectively. The data in Fig. 2B also reveal that a certain period of presence of ISP with cancer cells is mandatory to obtain clear-cut anti-cancer effects. Indeed, ISP must be added at its $\mathrm{IC}_{50}$ concentration for at least $24 \mathrm{~h}$ before displaying actual anti-cancer effects as revealed when treating human PC-3 prostate cancer cells with $2.5 \mu \mathrm{M}$ ISP (Fig. 2B). 
A
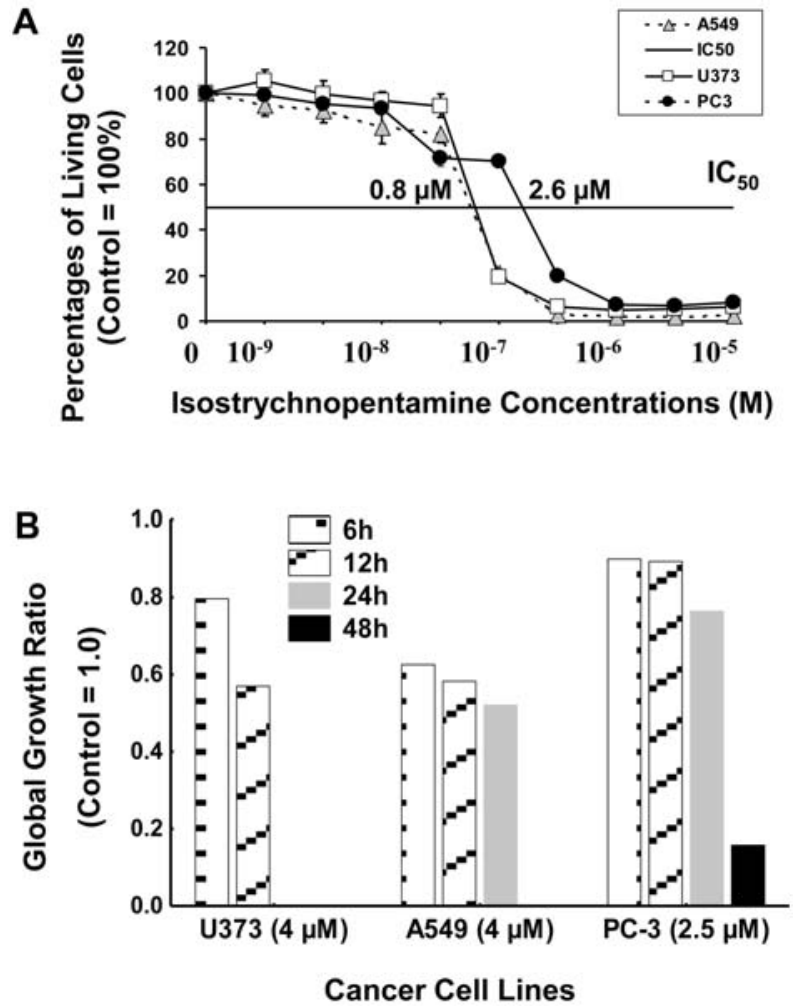

Figure 2. (A) Determination of the $\mathrm{IC}_{50}$ growth inhibitory concentration of ISP in 3 human cancer cell lines, i.e., the A549 NSCLC (gray triangles), the U373 GBM (the gray squares) and the PC-3 prostate cancer (the black dots) ones. ISP has been assayed from $1 \mathrm{nM}$ to $10 \mu \mathrm{M}$ with semi-log concentration increases. The experiments have been performed in hexaplicate and the data are presented as means \pm SEMs. (B) ISP-induced effects on the global growth ratio (number of cells after $6,12,24$ and $48 \mathrm{~h}$ divided by the number of cells at the beginning of the experiment) determined by means of computerassisted phase-contrast microscopy. Experiments were carried out in triplicate and the data are presented as median values.

ISP induces cytotoxic effects in human U373 GBM cells. The human U373 GBM cells resist many types of pro-apoptotic stimuli even when the pro-apoptotic compounds are analyzed at concentrations up to 10 -fold the compound-related $\mathrm{IC}_{50}$ concentrations $(17,18,23)$. Fig. 3 shows that $4 \mu \mathrm{M}$ ISP (i.e., $\sim 5 \mathrm{x} \mathrm{IC}_{50}$ concentration) is cytotoxic and kills nearly $100 \%$ of U373 GBM cells as quantitatively determined in Fig. 2B.

Is ISP a pro-apoptotic compound in U373 GBM and A549 NSCLC cells? We treated MCF-7 breast cancer cells with $1 \mu \mathrm{M}$ narciclasine for $72 \mathrm{~h}$ as a positive control $(15,18)$ labeled as ' $\mathrm{Ct}^{+}$' in Fig. 4A, which shows that ISP at its $\mathrm{IC}_{50}$ concentration $(\sim 1 \mu \mathrm{M})$ induces marked apoptotic features in U373 GBM cells (Fig. 4A), but not in A549 NSCLC cells (Fig. 4A). More than $80 \%$ of U373 GBM cells underwent early (gray bars; $50 \%$ ) and then late (vertical hatched bars; $\sim 30 \%$ ) apoptosis $48 \mathrm{~h}$ after having been treated with $1 \mu \mathrm{M}$ ISP ( $\mathrm{p}<0.01$ when compared to control; Fig. 4A). In sharp contrast, $<5 \%$ of A549 NSCLC cells underwent actual apoptosis (i.e., late apoptosis) when treated with ISP at its $\mathrm{IC}_{50}$ concentration ( $p>0.05$ when compared to control; Fig. 4A).

As explained in Discussion, it seems that part of the ISP-mediated proapoptotic effects in U373 GBM cells could be mediated by the ISP-induced transient decreases in $[\mathrm{ATP}]_{\mathrm{i}}$, which in addition is higher in control U373 GBM than in

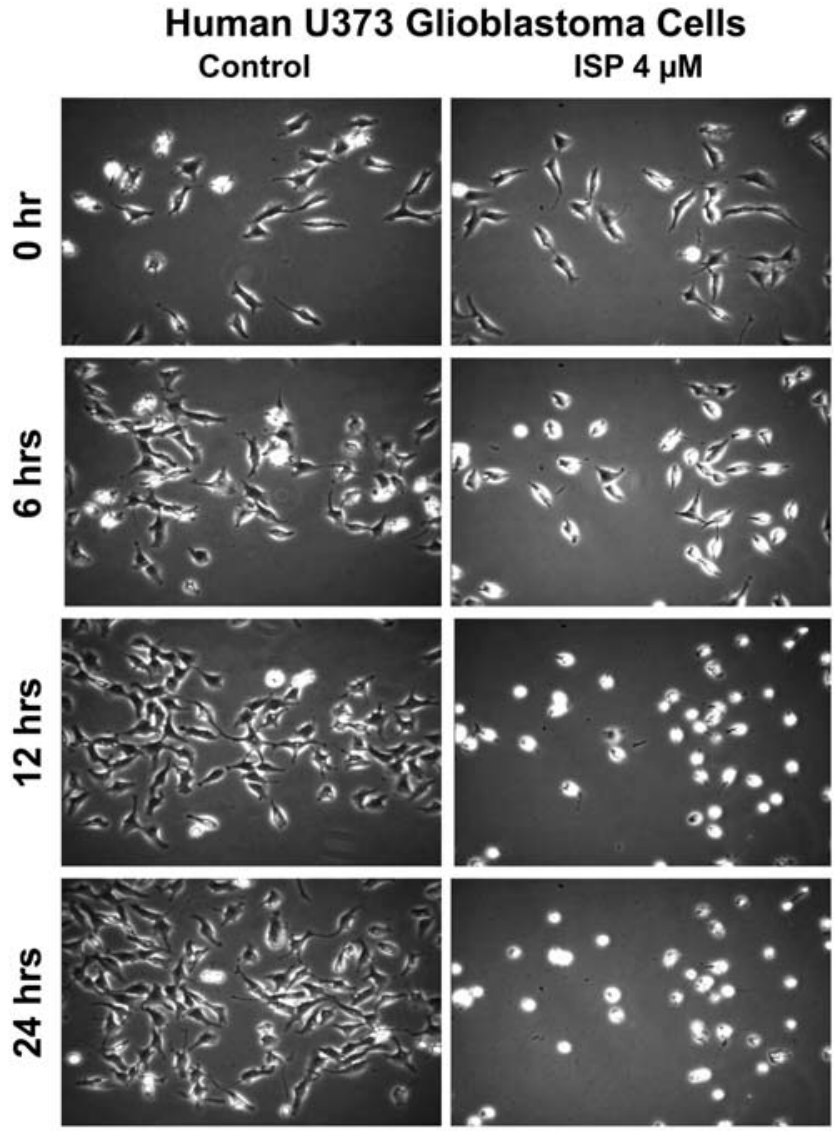

Figure 3. Morphological illustrations (computer-assisted phase-contrast microscopy) of $4 \mu \mathrm{M}$ ISP on the development of U373 GBM cell populations during $24 \mathrm{~h}$ of observation.

control A549 NSCLC cells (Fig. 4B), a feature that suggests that energy consumption markedly differs between U373 GBM and A549 NSCLC cells.

\section{Discussion}

The present study shows that ISP is able to induce apoptoticrelated cell death in human GBM cells (the U373 model) that display resistance to various pro-apoptotic stimuli $(17,18)$, while ISP is not able to induce apoptosis in human NSCLC cells (the A549 model) that are also displaying a certain level of resistance to various pro-apoptotic stimuli $(13,19)$. We have previously deciphered, at least partly, how ISP induces apoptosis in the human HCT-116 colon cancer cells (9), which displays sensitivity to pro-apoptotic stimuli $(24,25)$. ISP induces apoptosis in HCT-116 colon cancer cells as shown by the translocation of phosphatidylserine from the inner layer to the outer layer of the plasma membrane, chromatin condensation, DNA fragmentation, and caspase-3 and -9 activation (9). While ISP does not modify p53 expression in HCT-116 cancer cells, it induces p21 in a p53-independent manner (9). We also demonstrated that ISP did not affect the catalytic activity of human topoisomerases I and II (9), did not affect the polymerization or depolymerization of tubulin (unpublished data) and has no activity on kinases GSK3 and CDK5 (unpublished data) and we came to the conclusion that ISP promotes cancer cell death by a p53-independent apoptotic pathway (9). 
A

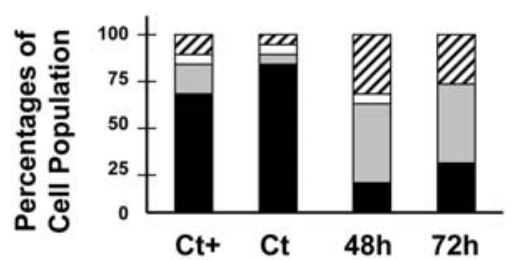

B

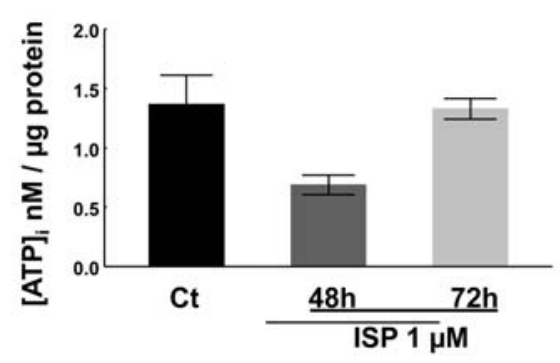

A549 NSCLC Cells
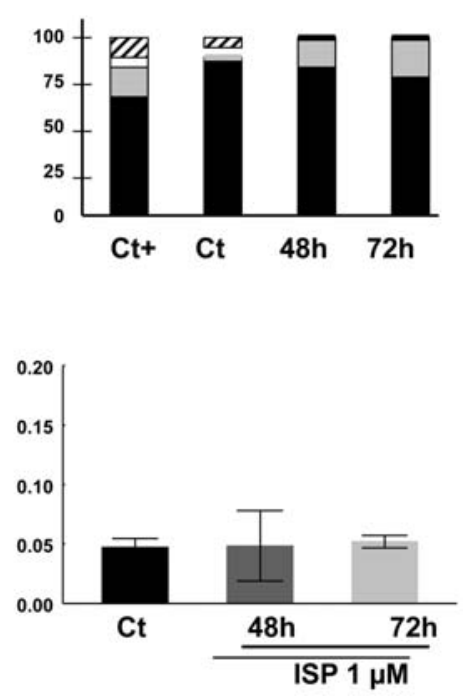

Figure 4. (A) Flow cytometry analyses of apoptosis versus necrosis induced by $1 \mu \mathrm{M}$ ISP for 48 and $72 \mathrm{~h}$ in U373 GBM and A549 NSCLC cells. Black bars correspond to normal cells [Annexin- $\mathrm{V}^{-}\left(\mathrm{AV}^{-}\right)$and propidium iodide- $(\mathrm{PI})$ ], gray bars to early apoptosis $\left(\mathrm{AV}^{+} / \mathrm{PI}^{-}\right)$, hatched vertical bars to late apoptosis $\left(\mathrm{AV}^{+} / \mathrm{PI}^{+}\right)$and open bars to necrosis $\left(\mathrm{AV}^{-} / \mathrm{PI}^{+}\right)$. Ct correspond to control cells, i.e., cells that have not been treated with ISP, while Ct ${ }^{+}$correspond to a positive control in which MCF-7 breast cancer cells have been treated with $1 \mu \mathrm{M}$ narciclasine for $72 \mathrm{~h}$. (B) Influence of $1 \mu \mathrm{M}$ ISP for 48 and $72 \mathrm{~h}$ in [ATP] in human U373 GBM and A549 NSCLC cells. The experiments were carried out in triplicate and the data are presented as means \pm SEMs.

The present study reveals that $[\mathrm{ATP}]_{\mathrm{i}}$ markedly differs between U373 GBM and A549 NSCLC cells (Fig. 4B) and we hypothesize that this is a crucial point in the fact that ISP induces apoptosis in U373 GBM cells, but not in A549 NSCLC ones (Fig. 4A).

Tumor cells display increased metabolic autonomy in comparison to non-transformed cells, taking up nutrients and metabolizing them in pathways that support growth and proliferation (26). Enhanced glycolysis is observed in most of cancer cells and tissues and is called as the Warburg effect (27). Enhanced glycolysis is essential both in the step of immortalization and transformation, as it renders cells resistant to oxidative stress and adaptive to hypoxic conditions, respectively (27). Enhanced glycolysis can be paralleled by reduced mitochondrial respiration (27), a feature that could occur in A549 NSCLC cells as compared to U373 GBM cells if we take into account the data of the present study. It must be kept in mind that besides their central roles in cell energy metabolism, mitochondria are also of special interest in cancer cell-related apoptosis (27). The precise mitochondrial alterations that underlie the increased dependence of cancer cells on aerobic glycolysis for energy generation have remained a mystery and recent evidence suggests that mitochondrial uncoupling, the abrogation of ATP synthesis in response to mitochondrial membrane potential, promotes the Warburg effect in leukemia cells, and may contribute to chemoresistance (28). Multiple myeloma, an incurable plasma cell malignancy, is also characterized by altered cellular metabolism and resistance to apoptosis (29). Glycolysis remains the critical pathway by which cancer cells meet their energy demands, not because of permanent transmissible alterations to the oxidative capacity of cells, but rather because of the inability of uncoupled mitochondria to generate ATP (28).

The fact remains that ISP exerts anti-cancer activities that are quite similar in both U373 GBM and A549 NSCLC cells (Fig. 1), a feature that strongly suggest that the main mechanism by which ISP exerts its anti-cancer effects does not relate to induction of apoptosis in cancer cells.

The data obtained in the present study with respect to ISP resemble those that we obtained previously with a mixture of steroidal saponins: balanitin-6 (28\%) and balanitin-7 (72\%) isolated from Balanites aegyptiaca kernels, a widely distributed African plant of medicinal interest containing a number of cytotoxic and cytostatic compounds. The balanitin- 6 and -7 mixture (henceforth referred to as bal6/7) has demonstrated appreciable anti-cancer effects in human cancer cell lines in vitro $(30) . \mathrm{Bal6} / 7$ demonstrated $\mathrm{IC}_{50}$ in vitro growth inhibitory concentrations in U373 GBM and A549 NSCLC cells quite similar for those observed here for ISP, i.e., $0.3 \mu \mathrm{M}$ (Bal6/7) versus $0.8 \mu \mathrm{M}$ (ISP) in A549 NSCLC cells and $0.5 \mu \mathrm{M}$ (Bal6/7) versus $0.8 \mu \mathrm{M}$ (ISP) in U373 GBM cells (30). Bal6/7 exerts its anti-cancer effects without inducing apoptotic cell death (30), but its in vitro anti-cancer activities result at least partly from depletion of $[\mathrm{ATP}]_{\mathrm{i}}$, leading in turn to major disorganization of actin cytoskeleton, ultimately resulting in the impairment of cancer cell proliferation (at the level of cytokinesis) and migration (30). In contrast to a number of natural products acting as anti-cancer agents, bal6/7 does not induce an increase in intra-cellular reactive oxygen species (30). In the present study, while we observed transient ISP-induced decreases in $[\mathrm{ATP}]_{\mathrm{i}}$ in U373 GBM cells, a feature that could explain (at least partly) ISP-induced proapoptotic effects in these U373 cells, we did not observe ISP-induced decrease in $[\mathrm{ATP}]_{\mathrm{i}}$ nor induction of apoptosis in A549 NSCLC cells. Thus, ISP-induced anti-cancer activity can lead to pro-apoptotic effects as a consequence, while apoptosis seems not to be the main cause by which ISP induces cancer cell death.

Altogether these data suggest that some plant-derived compounds, among which balanitines (30) and isostrychno- 
pentamine (the present study), are able to kill cancer cells that display certain levels of resistance to apoptosis at the same concentrations than cancer cells that are sensitive to apoptosis. The anti-cancer activity of these compounds is not mediated through the induction of apoptosis, even if proapoptotic features can be evidenced in some cancer cell lines. Balanitines and isostrychnopentamine are compounds that therefore merit further investigations in order to identify novel chemical leads aiming to overcome, at least partly, the resistance of metastatic cancers to apoptosis.

\section{Acknowledgements}

R.K. and M.F. are director of research and senior research associate from the Fonds National de la Recherche Scientifique (FNRS, Belgium), respectively. E.S.B. holds a grant from 'La Commission Universitaire pour le Dévelopement' (CUD/CIUF).

\section{References}

1. Savage P, Stebbing J, Bower M and Crook T: Why does cytotoxic chemotherapy cure only some cancers? Nat Clin Pract Oncol 6: 43-52, 2009.

2. Wilson TR, Johnston PG and Longley DB: Anti-apoptotic mechanisms of drug resistance in cancer. Curr Cancer Drug Targets 9: 307-319, 2009.

3. Simpson CD, Anyiwe K and Schimmer AD: Anoikis resistance and tumor metastasis. Cancer Lett 272: 177-185, 2008.

4. Cragg GM, Grothaus PG and Newman DJ: Impact of natural products on developing new anti-cancer agents. Chem Rev 109: 3012-3043, 2009.

5. Frederich M, Hayette MP, Tits M, De Mol P and Angenot L: In vitro activities of Strychnos alkaloids and extracts against Plasmodium falciparum. Antimicrob Agents Chemother 43 : 2328-2331, 1999.

6. Angenot L, Coune C and Tits M: Nouveaux alcalordes des feuilles du Strychnos usambarensis. J Pharm Beg 33: 11-23, 1978.

7. Tavernier D, Zhang W, Angenot L, Chierici-Tits M and Leclercq J: The structure of isostrychnopentamine, a bisindole monoterpene alkaloid from Strychnos usambarensis. Phytochemistry 26: 557-560, 1987.

8. Frédérich M, Tits M, Goffin E, Philippe G, Grellier P, De Mol P, Hayette MP and Angenot L: In vitro and in vivo antimalarial properties of isostrychnopentamine, an indolomonoterpenic alkaloid from Strychnos usambarensis. Planta Med 70: 520-525, 2004.

9. Frédérich M, Bentires-Alj M, Tits M, Angenot L, Greimers R, Gielen J, Bours V and Merville MP: Isostrychnopentamine, an indolomonoterpenic alkaloid from Strychnos usambarensis, induces cell cycle arrest and apoptosis in human colon cancer cells. J Pharmacol Exp Ther 304: 1103-1110, 2003.

10. Ceruti S, Mazzola A and Abbracchio MP: Resistance of human astrocytoma cells to apoptosis induced by mitochondria-damaging agents: possible implications for anticancer therapy. J Pharmacol Exp Ther 314: 825-837, 2005.

11. Lefranc F, Brotchi J and Kiss R: Possible future issues in the treatment of glioblastomas: special emphasis on cell migration and the resistance of migrating glioblastoma cells to apoptosis. J Clin Oncol 23: 2411-2422, 2005.

12. Sarkaria JN, Kitange GJ, James CD, Plummer R, Calvert H, Weller M and Wick W: Mechanisms of chemoresistance to alkylating agents in malignant glioma. Clin Cancer Res 14: 2900-2908, 2008.

13. Mathieu A, Remmelink M, D'Haene N, Penant S, Gaussin JF, van Ginckel R, Darro F, Kiss R and Salmon I: Development of a chemoresistant orthotopic human non-small cell lung carcinoma model in nude mice: analyses of tumor heterogeneity in relation to the immunohistochemical levels of expression of cyclooxygenase-2, ornithine decarboxylase, lung-related resistance protein, prostaglandin E synthetase, and glutathioneS-transferase-alpha (GST)-alpha, GSY-mu and GST-pi. Cancer 101: 1908-1918, 2004.
14. Fenell DA: Caspase regulation in non-small cell lung cancer and its potential for therapeutic exploitation. Clin Cancer Res 11: 2097-2105, 2005

15. Dumont $\mathrm{P}$, Ingrassia L, Rouzeau S, Ribaucour F, Thomas S, Roland I, Darro F, Lefranc F and Kiss R: The Amaryllidaceae isocarbostyril narciclasine induces apoptosis by activation of the death receptor and/or mitochondrial pathways in cancer cells but not in normal fibroblasts. Neoplasia 9: 766-776, 2007.

16. Mijatovic T, van Quaquebeke E, Delest B, Debeir O, Darro F and Kiss R: Cardiotonic steroids on the road to anti-cancer therapy. Biochim Biophys Acta 1776: 32-57, 2007.

17. Lefranc F, Mijatovic T, Kondo Y, Sauvage S, Roland I, Debeir O, Krstic D, Vasic V, Gailly P, Kondo S, Blanco G and Kiss R: Targeting the alpha-1 subunit of the sodium pump to combat glioblastoma cells. Neurosurgery 62: 211-222, 2008.

18. Ingrassia L, Lefranc F, Dewelle J, Pottier L, Mathieu V, SpieglKreinecker S, Sauvage S, El Yazidi M, Dehoux M, Berger W, van Quaquebeke E and Kiss R: Structure-activity relationship analysis of novel derivatives of narciclasine (an Amaryllidaceae isocarbostyril derivative) as potential anticancer agents. J Med Chem 52: 1110-1114, 2009.

19. Mijatovic T, Mathieu V, Gaussin JF, De Nève N, Ribaucour F, van Quaquebeke E, Dumont P, Darro F and Kiss R: Cardenolideinduced lysosomal membrane permeabilization demonstrates therapeutic benefits in experimental human non-small cell lung cancers. Neoplasia 5: 402-412, 2006.

20. De Hauwer C, Camby I, Darro F, Migeotte I, Decaestecker C, Verbeek C, Danguy A, Pasteels JL, Brotchi J, Salmon I, van Ham P and Kiss R: Gastrin inhibits motility, decreases cell death levels and increases proliferation in human glioblastoma cell lines. J Neurobiol 37: 373-382, 1998.

21. Debeir O, Van Ham P, Kiss R and Decaestecker C: Tracking of migrating cells under phase-contrast videomicroscopy with combined mean-shift processes. IEEE Trans Med Imaging 24: 697-711, 2005.

22. Lefranc F, Sauvage S, Van Goietsenoven G, Mégalizzi V, Lamoral-Theys D, Debeir O, Spiegl-Kreinecker S, Berger W, Mathieu V, Decaestecker C and Kiss R: Narciclasine, a plant growth regulator, activates Rho and stress fibers in glioblastoma cells. Mol Cancer Ther 8: 1739-1750, 2009.

23. Branle F, Lefranc F, Camby I, Jeuken J, Geurts-Moespot A, Sprenger S, Sweep F, Kiss R and Salmon I: Evaluation of the efficiency of chemotherapy in in vivo orthotopic models of human glioma cells with and without $1 \mathrm{p} 19 \mathrm{q}$ deletions and in C6 rat orthotopic allografts serving for the evaluation of surgery combined with chemotherapy. Cancer 95: 641-655, 2002.

24. Palozza P, Sestito R, Picci N, Lanza P, Monego G and Ranelletti FO: The sensitivity to beta-carotene growth-inhibitory and proapoptotic effects is regulated by caveolin-1 expression in human colon and prostate cancer cells. Carcinogenesis 29: 2153-2161, 2008.

25. Hu CM and Chang ZF: Synthetic lethality by lentiviral short hairpin RNA silencing of thymidylate kinase and doxorubicin in colon cancer cells regardless of the p53 status. Cancer Res 68: 2831-2840, 2008

26. De Berardinis RJ, Sayed N, Ditsworth D and Thompson CB: Brick by brick: metabolism and tumor cell growth. Curr Opin Genet Dev 18: 54-61, 2008.

27. Kondoh H: Cellular life span and the Warburg effect. Exp Cell Res 314: 1923-1928, 2008.

28. Samudio I, Fiegl M and Andreeff M: Mitochondrial uncoupling and the Warburg effect: Molecular basis for the reprogramming of cancer cell metabolism. Cancer Res 69: 2163-2166, 2009.

29. Shanmugam M, McBrayer SK, Qian J, Raikoff K, Avram MJ, Singhal S, Gandhi V, Schumacker PT, Krett NL and Rosen ST: Targeting glucose consumption and autophagy in myeloma with the novel nucleoside analogue 8-amino-adenosine. J Biol Chem (In press)

30. Gnoula C, Mégalizzi V, De Nève N, Sauvage S, Ribaucour F, Guissou P, Duez P, Dubois J, Ingrassia L, Lefranc F, Kiss R and Mijatovic T: Balanitin-6 and -7: Diosgenyl saponins isolated from Balanites aegyptiaca Del. display significant anti-tumor activity in vitro and in vivo. Int J Oncol 32: 5-15, 2008. 